\title{
ESCENARIOS LINGÜÍSTICOS EMERGENTES EN LA FRONTERA TIJUANA-SAN DIEGO
}

\author{
Emergent linguistic scenarios at the border region Tijuana-San Diego
}

\author{
David Guadalupe Toledo Sarracino*1 \\ Laura García-Landa**
}

\begin{abstract}
RESUMEN
La presente contribución es un primer estudio exploratorio para comprender de qué manera las dinámicas de migración en el desarrollo económico y social actual en Baja California se están integrando al mosaico lingüístico existente en Tijuana, como resultado del desarrollo económico y del crecimiento demográfico de la población migrante en la región. Esta primera descripción podría servir como punto de partida para otros estudios y, específicamente, para la política y planificación lingüística en la región fronteriza (Tijuana-San Diego) en un principio, pero también para otras regiones que experimentan migraciones masivas de manera acelerada. Este estudio de tipo cualitativo descriptivo señala algunos ejes específicos sobre la reciente migración de Centroamérica y el Caribe en Baja California, así como las necesidades lingüísticas de migrantes deportados o retornados en estos últimos meses de 2017, debido a la rigurosa política migratoria del presidente de los Estados Unidos, Donald J. Trump. Por otro lado, se describe la realidad de los mexicanos repatriados frente a esta situación. Los resultados del presente estudio revelan escenarios lingüísticos emergentes que se viven en la Frontera en general y, más específicamente, sobre el papel que juegan las lenguas en los nuevos migrantes de Baja California.
\end{abstract}

Palabras clave: frontera, migración, escenarios lingüísticos, lengua, Tijuana-San Diego.

\begin{abstract}
The following article is the first exploratory study, which has a main objective to understand the dynamics of migration in the current economic and social matters in Baja California; due to economic development and the demographic population grows in Tijuana a new linguistic mosaic has been incorporated.

This first description will support other studies, specifically in the field of language planning and language policy at the border region (Tijuana-San Diego), as a beginning but at the same time other regions that experiment massive and accelerated migration. This is a qualitative-descriptive study that describes specific axes regarding the recent migration from Central America and the Caribbean in Baja California, as well as linguistic needs that the deported immigrants in the last months of 2017 due to the rigorous migration policy of President of the Unites States of America, Donald J. Trump. On the other hand, this study describes the reality that deported and returned immigrants face toward this current situation. The result of this study reveals the linguistic emergence scenarios of immigrants who live at the border region and specifically the role of the languages in the new immigrants of Baja California.
\end{abstract}

Key Words: frontier, migration, linguistic scenario, language, Tijuana-San Diego.

\section{Antecedentes}

\footnotetext{
1 * Universidad Autónoma de Baja California. Profesor investigador en la Facultad de Idiomas. México. Correo electrónico: dtoledo@uabc.edu.mx

** Universidad Nacional Autónoma de México. Profesora titular en la Escuela Nacional de Lenguas, Lingüística y Traducción. México. Correo electrónico: garlanster@gmail.com
} 
A partir del 2016 iniciaron los movimientos migratorios de la población haitiana a México, precisamente Baja California es el estado que presenta mayor migración haitiana en el país. El Gobierno Federal y Estatal ha ofrecido ayuda a través de organizaciones no gubernamentales [ONG] en apoyo a los Estados Unidos para detener a la migración que desea ingresar a este país con el estatus de ayuda humanitaria. De acuerdo con Loza (2017), las ciudades de Tijuana, Mexicali y Tecate cuentan con más de 20 albergues, los cuales tienen una capacidad insuficiente, ya que se recibe una cantidad superior a los 3000 migrantes haitianos y africanos, con lenguas como el criollo y el francés. Quienes tuvieron la oportunidad de estar en misiones de trabajo en Brasil, hablan portugués brasileño; actualmente, se ven forzados a aprender español, con el afán de permanecer en Tijuana y encontrar mejores oportunidades laborales. Tijuana se ha convertido, por tanto, en una ciudad de espacio fronterizo donde convergen lenguas distintas al inglés y al español.

El gobierno del estado de Baja California ha desarrollado una iniciativa de apoyo a las comunidades migrantes emergentes, las cuales han resultado insuficientes. Los albergues desarrollan estrategias de apoyo a esas minorías que se han ubicado en las ciudades como Tijuana, Mexicali y Tecate. Para esos caribeños y africanos estar en México representa una oportunidad para lograr una mejor calidad de vida. Tijuana tiene zonas de clase baja donde viven los new comers caribeños, que lejos de continuar los intentos de cruzar a los Estados Unidos con una visa humanitaria, sus deseos se ven truncados por las nuevas iniciativas migratorias de la Era Trump. La única esperanza que poseen es la de permanecer en México, legalizar su estatus migratorio en Tijuana y encontrar un trabajo legal.

Las políticas migratorias establecidas por el presidente de Donald J. Trump se han endurecido y la esperanza de obtener una visa humanitaria se torna difícil. Loza (2017) señala que las familias han decidido internarse en los Estados Unidos y permanecer detenidos sin hablar inglés ni español. Es decir, estas familias de haitianos están en espera de una oportunidad para un estatus migratorio en este país. Se requiere de intérpretes para establecer comunicación con los cientos de familias que se encuentran en proceso de ser deportados.

Baja California es el espacio geográfico que brinda oportunidades laborales a migrantes de distintas partes del país, y de países extranjeros, tales como: Honduras, Nicaragua, Guatemala, Belice. Todos estos migrantes al establecerse en Baja California deben contender por la obtención de vivienda y un espacio laboral que les permita mejorar 
sus condiciones de vida. Además, se identifican dos comunidades lingüísticas que han crecido en número e importancia en Tijuana: la comunidad de haitianos y los mexicanos repatriados.

\subsection{Comunidades lingüísticas emergentes en Tijuana}

\subsubsection{La comunidad haitiana}

En 2016, a partir del flujo atípico de haitianos en la frontera Tijuana-San Diego, se ha solicitado al Gobierno mexicano apoyo para que esta comunidad se establezca de manera legal. Es decir, con la suspensión de las visas humanitarias para haitianos desde la administración del presidente Barack Obama, estar varados en esta frontera ha sido la principal cuestión para que esta comunidad se establezca en Tijuana y Mexicali. Según informes del Comité Ciudadano en Defensa de los Naturalizados y Afromexicanos [CCDNA] (2017), el inicio de la cancelación de visas humanitarias para ciudadanos de Haití fue el argumento que había concluido el estado de emergencia para la isla del Caribe.

De acuerdo con el estudio realizado por El Universal (2016), el trayecto que más de 3000 haitianos han recorrido para atravesar México y Centroamérica es una jornada que muestra carestía, sufrimiento, abuso por parte de los traficantes de personas que cobran más de 5000 dólares para internarlos en la frontera y llevarlos, finalmente, al "sueño americano".

De lo expuesto, se deduce que la necesidad de aprender español para estas personas es urgente. Para estos hablantes, la familiarización con la lengua es una herramienta para la sobrevivencia. Resulta imprescindible que se enseñe español a los migrantes haitianos y africanos para que puedan sobrevivir en la frontera. Por otro lado, el aprender inglés representaría un valor agregado en sus aspiraciones y posibilidades de mejoras laborales en la frontera. Cualquier habitante de Tijuana puede encontrar a estos migrantes en los diversos cruceros de la ciudad ofreciendo servicios como franeleros o "viene- viene", 2 así como desempeñando actividades de meseros, cocineros, carpinteros, albañiles, lava carros, personal de limpieza, etc., en locales establecidos.

Con base en los registros de la Comisión de Ayuda Humanitaria [CAH] (2016), un $40 \%$ de los migrantes registrados en los albergues cuentan con estudios de primaria y

\footnotetext{
${ }^{2}$ Trabajo informal por el que una persona vigila la salida y entrada de un auto que está estacionado en vía pública, a cambio de algunas monedas.
} 
secundaria. Un $5 \%$ cuenta con estudios de preparatoria y universidad; lo que representa que en poco tiempo, si cuentan con los espacios propicios, podrán acceder a educación superior. El resto no tiene estudios de primaria. Corresponde al Estado y a las sociedades civiles apoyar a esta comunidad en la integración de estos migrantes a los diferentes sectores sociales, económicos, educativos, culturales, de acuerdo con sus formación educativa y necesidades, ya que dentro de esta comunidad también hay personas con perfiles formativos diversos: plomeros, albañiles, estilistas, abogados, médicos, profesores y enfermeras.

Según Loza (2017), en los comedores comunitarios y albergues han iniciado a impartirse clases de español como lengua de acceso o de sobrevivencia para que esos new comers tengan la oportunidad de obtener un empleo digno o convertirse en vendedores ambulantes con el limitado nivel que su español les permite. Sin embargo, los esfuerzos de esa comunidad han sido insuficientes, dado que se requiere de apoyo de las instituciones gubernamentales y de asociaciones civiles para agilizar su permanencia en Tijuana bajo un estatus de permiso de permanencia humanitaria.

La lengua meta a aprender y su estatus migratorio son los principales obstáculos para que esta minoría compita e incursione por espacios laborales formales y estables junto a otros migrantes.

\subsubsection{El portugués por hablantes haitianos}

En la actualidad, hay una realidad que afronta esta lengua en la región. A partir de la migración haitiana a la localidad, y de acuerdo con el proyecto Archivo con testimonios de los Migrantes, desarrollado por el Colegio de la Frontera Norte (2016), existe un grupo de 1057 haitianos registrados, de los cuales 200 de ellos hablan portugués, debido a que participaron en misiones de jornadas laborales en Brasil. Esto los hace tener una lengua adicional para fines laborales. A pesar de que esta no es la lengua de acceso para mejores oportunidades de trabajo, la aproximación lingüística ha funcionado como medio de comunicación. Es decir, que se ha desarrollado un pidgin entre el español, el portugués y el criollo en este espacio fronterizo. Aunque la mayor parte de ellos subsisten en México, todavía algunos, si no es que la mayoría, desean tener acceso a los Estados Unidos, lo cual convierte a la lengua inglesa en una herramienta para oportunidades laborales y de mejora económica y, por ende, se inclina hacia una micro-comunidad plurilingüe. 


\subsubsection{Los mexicanos repatriados o retornados}

Según Couza (2014), el número de mexicanos residentes fuera de México es equivalente a 12178173 mexicanos, según el censo de 2012. Dentro de esta cifra, se encuentran los migrantes, desplazados, deportados, retornados y extraditados, lo cual convierte al país con mayor número de migrantes de comunidades latinas y caribeñas hacia los Estados Unidos.

En sí, el flujo migratorio de todos aquellos mexicanos indocumentados o de estatus inestable, que fueron deportados durante el gobierno de Obama y las estrictas medidas de migración del gobierno de Trump, imperantes en la actualidad, impiden continuar viviendo en Estados Unidos. Por tanto, de alguna manera, el espacio fronterizo Tijuana-San Diego ha acogido a todas estas personas, convirtiéndose así en el lugar indicado para vivir:

El gobierno estadounidense clasifica dos tipos de deportados: los removidos (removals) mediante una orden de deportación después de años de vivir en Estados Unidos y los retornados "voluntariamente" (returns) que son capturados en su intento de ingresar a territorio norteamericano. El uso del concepto "repatriados" por parte del gobierno mexicano para definir a todos los expulsados a territorio nacional es criticado por Padilla (2012) porque oculta características de vida de las personas que son obligadas a salir de Estados Unidos (Ibarra, 2016, p. 43).

Tanto los removidos, retornados o repatriados viven una dinámica social, cultural y lingüística a la que no han pertenecido durante años, extraña y ajena a su experiencia de vida, lo cual muchas veces los pone en desventaja laboral frente a los mexicanos no emigrados. Sin embargo, la lengua inglesa los coloca por encima de ellos, cuando el inglés deviene requisito laboral y los migrantes retornados o binacionales pueden incorporarse fácilmente en esas posiciones:

La frontera mexicana con Estados Unidos se convirtió en el destino preferido de las migraciones internas y destaca que Tijuana pasó de tener 25 mil habitantes en los cuarentas a alcanzar el millón en los noventas. En el periodo de las expulsiones a estudiar las condiciones de empleo en Baja California, donde se ubica Tijuana, 
eran poco favorables para la llegada de los deportados, también migrantes, pero internacionales (Ibarra, 2016, p. 52).

Aparte de estos mexicanos que están de regreso a su país natal, con certeza vendrán mexicanos que poseerán destrezas y habilidades para el trabajo, además de un buen nivel de dominio del inglés; pero con deficiencias en el español, problemas para afrontar diversas situaciones socioeconómicas y adaptarse a una nueva vida en México.

Esta información aludida ha sido documentada por Anderson (2013), quien menciona que de 2000 a 2010 ha habido un incremento del $116 \%$ de call centers en México, los cuales reclutan migrantes retornados con un nivel educativo igual o superior a la preparatoria. No obstante, Zúñiga (2013) advierte de la migración de niños y adolescentes trasnacionales o binacionales, quienes se enfrentan al fuerte conflicto de no encontrar políticas educativas que den respuesta a sus necesidades particulares de transición de un sistema educativo a otro, y en el que tanto la enseñanza de materias curriculares en inglés, como el desarrollo de la alfabetización en lengua castellana, es fundamental para preparar a las futuras generaciones de estudiantes migrantes del país.

Lo anterior pone en evidencia la relevancia de atender de manera urgente esta problemática en el ámbito de la educación básica, enfatizando las consecuencias catastróficas que podrían resultar al ignorar este llamado. Ya Smith (2006) divisaba algunas posibilidades de integración de los estudiantes transnacionales para mantener el bilingüismo transnacional español-inglés, pero también los impedimentos, muy asociados principalmente a los estereotipos hacia el estigma de los migrantes retornados y su sociolecto, bajo la mítica y romántica idea de la existencia de una única variante de prestigio, la británica. Tanto Smith (2006) como Zúñiga, siete años después, aciertan sobre la necesidad de sensibilizar a los profesores y a la comunidad en general acerca de las actitudes y creencias sobre los migrantes retornados para su inserción a la vida escolar y, se añade, en un futuro al sector laboral. Smith (2006) proponía desarrollar fondos de conocimiento lingüístico, los cuales consistían en lo siguiente:

En las escuelas y comunidades con altas concentraciones de retornados, los profesores interesados deben tener todo el apoyo necesario para aprender más sobre los conocimientos lingüísticos y culturales de sus estudiantes. Dicha preparación debe incluir cursos de formación en la adquisición de L1 y L2, además de cursos sobre los aspectos psicológicos y sociolingüísticos de la adquisición. 
Asimismo deberán cursar educación bilingüe, con énfasis en cómo reconocer los recursos lingüísticos de los transnacionales y cómo trabajar con estos recursos e integrarlos en el salón de clase, tanto en las asignaturas de contenido como en las de inglés y español (...), una aplicación más ambiciosa, pero no por eso menos interesante, podría ser el reclutamiento de retornados como candidatos a los programas de educación en México. Se sabe que los niños aprenden mejor cuando tienen interacciones con profesores y otros adultos con los quienes se identifiquen (Smith, 2006, p. 436).

Lo dicho se hace mucho más urgente cuando se observa la conformación migratoria compleja de Tijuana.

\subsubsection{Tijuana, la migración y las lenguas}

De acuerdo con Toledo Sarracino y Leopoldino (2018), Tijuana es la ciudad más desarrollada del noroeste de México con un total de 1693 923,00 habitantes según el censo del Instituto Nacional de Estadística Geografía e Informática [INEGI] (2015). Es la ciudad con mayor desarrollo económico en el estado de Baja California y colinda al norte con el condado de San Diego, California. Por su parte, San Diego es uno de los puertos navales más importantes de Estados Unidos tiene un total de 1307 402,00 habitantes. En Tijuana, existen por lo menos siete agrupaciones lingüísticas consolidadas de lenguas extranjeras como el inglés, el francés, el chino cantonés, el chino mandarín, el coreano, el japonés, el alemán y el portugués; a partir de finales del 2016, se suma el criollo.

Además, con base en los registros del Instituto Nacional de las Lenguas Indígenas (INALI, 2008) publicados a través del Diario Oficial de la Federación, llamado Catálogo de las Lenguas Indígenas Nacionales: Variantes Lingüísticas de México con sus autodenominaciones y referencias geo-estadísticas, en Baja California se encuentran representadas ocho familias lingüísticas indoamericanas que pertenecen total $o$ parcialmente al territorio mexicano, tales como: mayo, yaqui, mixteco, zapoteco, otomí, kiliwa, cucapá, etc. Así como la variación dialectal del español como resultado de la migración interna por cuestiones laborales en los últimos cinco años. Esto hace de Tijuana una frontera con una amplia gama de lenguas indígenas nacionales que interactúan y se 
entrelazan para comunicarse, mostrando un mosaico lingüístico particular de esta frontera.

\subsubsection{El criollo y el español como lengua de trabajo en la Frontera}

La variante dialectal del francés criollo hablado en Haití es una lengua que se agrega a la gama de lenguas habladas de la frontera Tijuana-San Diego. Dadas las particularidades de la lengua, se ha podido tener acercamiento con la comunidad a través de los programas de servicio social comunitario de los alumnos aprendientes del francés de la UABC.

Sin embargo, el español es la lengua de facto determinada por el estado. Para Terborg, García Landa y Moore (2006) esta lengua está determinada por el estado para todos los fines, puesto que el $90 \%$ de la población habla esa lengua, ya sea por sus funciones históricas o legislativas, y es la lengua franca entre los hablantes de lengua indígena. Es decir, al igual que las comunidades autóctonas que llegan a Tijuana, estos migrantes o new comers deben hablar español para fines de sobrevivencia, laborales o de interacción social.

Por otro lado, son acciones mínimas para mitigar la necesidad de comunicarse, por lo tanto, aprender el español es imperante. Según el proyecto Archivo con testimonios de los migrantes desarrollado por el Colegio de la Frontera Norte [COLEF] (2016), y con base en los reportes hasta finales del año 2016, se habían contabilizado 1057 haitianos, 259 de origen mexicano y 11 de otros países centroamericanos.

Los migrantes en esta frontera norte han conformado una comunidad integrada en los 13 albergues en Tijuana. Ante ello, las organizaciones no lucrativas solicitan apoyo a las instituciones gubernamentales y no gubernamentales para pedir alimentos, productos de higiene personal y productos para cubrir todas las necesidades básicas. Al igual, dentro de esos albergues se han integrado grupos de apoyo para impartir clases de español con propósitos de enseñar competencias básicas de una lengua de sobrevivencia.

\subsubsection{El inglés como oportunidad económica}

Otra lengua que debe ser observada con detenimiento es el inglés. Con base en los informes del Instituto Nacional de Migración [INAMI] en México, durante el 2017 han sido deportados alrededor de 12447 connacionales que vivían de forma ilegal en Estados Unidos. Según el INAMI (2017), la cifra de deportados es mayor a las estadísticas en los periodos de 2016 durante el gobierno de Barack Obama; lo que representa que el nivel de 
deportados ha incrementado significativamente en el 2017 a partir de las políticas migratorias del presidente de los Estados Unidos, Donald J. Trump. Debido al tiempo que estas personas han estado expuestos al idioma inglés, lo han aprendido y el conocimiento que poseen les permite demostrar una competencia suficiente.

El inglés es la lingua franca para los habitantes fronterizos, pero también es lengua de facto por el hecho de estar en la frontera. Hablar inglés representa acceder a las mejores oportunidades laborales por su colindancia con San Diego, California. El ciudadano fronterizo que habla inglés sabe que es sinónimo de mejoría laboral. El inglés sigue representando un valor agregado para quienes transitan, trabajan, estudian y tienen un estilo de vida de un ciudadano fronterizo. Este idioma tiene la misma posición de uso que el español, dado que ambas lenguas son lenguas determinantes para tener una vida binacional. En un entorno en el que Tijuana y San Diego son vistos como una región binacional o mega-región económica importante para sus Estados (García Landa, en preparación).

Otro grupo de flujo migratorio lo constituyen los repatriados o retornados en Tijuana. Para ellos, el inglés es una habilidad lingüística no certificada o reconocida para mejorar la búsqueda de empleo en México y, en especial, en la frontera México-Estados Unidos:

Los deportados que llegan a Tijuana y área conurbada están encontrando empleo en una diversidad de segmentos del mercado laboral, sin embargo, hay nichos que han aprovechado sus competencias laborales como son los call centers. Las empresas encontraron en esta población la oportunidad de contratar a personas que además de dominar el idioma inglés tienen la experiencia de vivir en Estados Unidos (Ibarra, 2016, p. 57).

La oportunidad laboral para los deportados, repatriados o retornados a México es en el sector maquiladora y en los clúster de call centers en Tijuana. Otro nicho de oportunidad es en las empresas de importación-exportación donde el inglés es requerido. Es pertinente puntualizar que quienes poseen estudios de preparatoria han visto como oportunidad de trabajo la enseñanza del inglés con la respectiva formación docente o trabajar como brokers en las casas aduaneras. El futuro es crítico para este tipo de ciudadanos, debido a que sus condiciones tendrán que incorporarse poco a poco a una vida laboral en México. 
Para esta investigación, se ha establecido como objetivo mostrar las primeras aproximaciones sobre los escenarios lingüísticos emergentes en la frontera Tijuana-San Diego, además de dar a conocer las nuevas dinámicas de migración en el desarrollo económico y social en Baja California a partir de la nueva integración de un mosaico multicultural y lingüístico existente en Tijuana. Para ello, se responderán algunas interrogantes establecidas en este trabajo:

1. ¿Qué experimenta esta zona fronteriza a partir de migraciones masivas de forma acelerada?

2. ¿Cómo se describe el mosaico lingüístico en esta frontera a partir de la nueva migración?

3. ¿Cuál es el futuro de la política y planificación lingüística de esta frontera a partir de este crecimiento acelerado de migrantes?

Estas preguntas se responderán a partir de la metodología establecida en el estudio y las aproximaciones que se identificaron a partir de los resultados de los encuestados.

\section{Metodología del estudio}

Debido a las características del estudio, se optó por una aproximación cualitativadescriptiva que diera cuenta de los escenarios lingüísticos emergentes en la frontera Tijuana-San Diego. En este sentido, se diseñó un cuestionario dirigido a los responsables de las casas del migrante establecidas en la Ciudad de Tijuana, B. C., México. Se tomó como base el Proyecto Salesiano de Tijuana, desayunador Padre Chava en Tijuana, Baja California, México y las instalaciones del INAMI. Esta organización no gubernamental sin fines de lucro brinda alimento y apoyo al migrante para repatriarse a sus lugares de origen o legalizar su permanencia en Tijuana.

El cuestionario cuenta de diez incisos para recabar datos de los migrantes y obtener información relevante para el estudio de escenarios lingüísticos emergentes en la frontera Tijuana-San Diego. En dicha encuesta contempla criterios como edad, lugar de origen, lenguas que domina, tiempo de radicar en la ciudad de Tijuana, situación legal en el país, conocer antecedentes lingüísticos sobre el español y otras lenguas, así como los propósitos que tienen como migrantes al permanecer en la frontera.

Dicho instrumento también recopiló los antecedentes linguiísticos, intereses y datos sobre los oficios y profesiones de los migrantes; además de conocer las intenciones 
de informantes sobre su permanencia en la ciudad. En las instalaciones del lugar donde se realizó la recolección de los datos se habían registrado 156 migrantes (mexicanos, hondureños, salvadoreños y haitianos). Para los fines de este estudio se utilizarán los resultados de cien encuestados, ya que fueron las personas que accedieron a responder el cuestionario.

\section{Resultados}

A continuación, se muestran los resultados obtenidos a partir de la encuesta denominada "Estudio: Escenarios lingüísticos emergentes en la frontera Tijuana-San Diego".

En la Figura 2.1, se muestra que la edad del $50 \%$ de las personas es entre 30 y 40 años; un $20 \%$ entre los 18 y 20 años; un $17 \%$ entre 21 y 30 años; y un $13 \%$ en edad de 40 a 50 años. Lo que significa que todos los migrantes que fueron encuestados se encuentran en una edad productiva para realizar actividades laborales en la frontera.

\section{Edad}

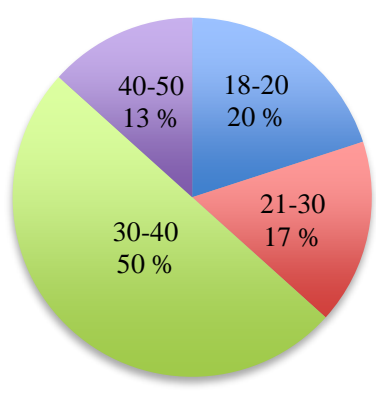

Figura 2.1. Edad

En la Figura 2.2, el $58 \%$ pertenecen al sexo masculino y el $42 \%$ al sexo femenino. Es decir, que de la muestra de este estudio, la mayoría son del sexo masculino. 


\section{Sexo}

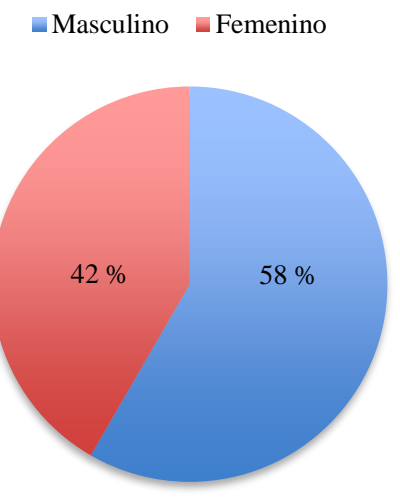

Figura 2.2. Sexo

En la Figura 2.3, se muestra que el $69 \%$ de los migrantes son hablantes del criollo, lo cual significa que son de nacionalidad haitiana; un $24 \%$ son hablantes del español provenientes de Centroamérica. En el rubro del $7 \%$, se integran los mexicanos que nacieron en México, quienes de niños cruzaron la frontera, vivieron de forma ilegal con sus padres, se desarrollaron en un entorno monolingüe (inglés), carecen de documentos de residencia permanente para vivir en Estados Unidos y argumentan que su primera lengua es el inglés.

\section{Lengua materna}



Figura 2.3. Lengua materna

En la Figura 2.4, se presenta el tiempo que los migrantes tienen viviendo en Tijuana. El $62 \%$ tienen de radicar en la ciudad de 1 a 6 meses, el $31 \%$ alrededor de 12 meses, el $6 \%$ de 2 a 5 años y el $1 \%$ de 1 a 3 años. Lo que indica que en el porcentaje más alto 
se centra en aquellos residentes haitianos y centroamericanos, dentro de estos, el menor porcentaje de los encuestados se reserva para los migrantes mexicanos.

\section{Tiempo de radicar en Tijuana}

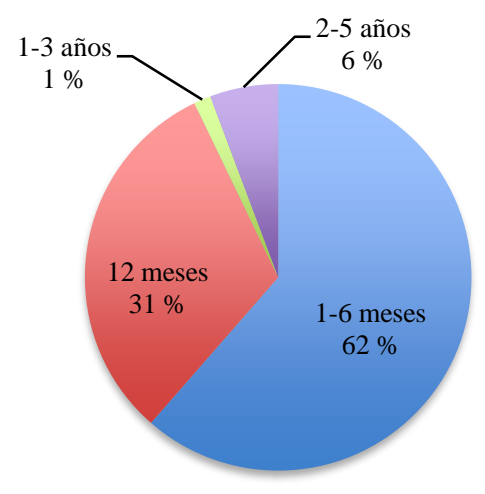

Figura 2.4. Tiempo de radicar en la ciudad

Debido a las particularidades del mosaico lingüístico de esta frontera, se les preguntó a los informantes sobre la importancia que le dan a las lenguas para acceder a mejores escenarios laborales y estilo de vida más decoroso. Ante ello, como se observa en la Figura 2.5, estos nombraron en primer lugar el español, en segundo lugar al inglés, en tercer lugar al chino mandarín, en cuarto lugar al portugués, en quinto a otras lenguas $y$, finalmente, al francés. Lo que manifiesta que los hablantes haitianos residentes en Tijuana ven como prioridad aprender español, dado que es una lengua que les permitirá laborar e interactuar con fines de sobrevivencia. Este idioma brinda a los migrantes una mejor posición en el desarrollo económico y social de la frontera. Además, el inglés ocupa el segundo lugar, lo cual representa para el migrante haitiano una oportunidad económica con fines de acceder a mejores escenarios laborales.



Figura 2.5. Posicionamiento de las lenguas en la frontera 
Por otro lado, es necesario potencializar el papel que juega el español para extranjeros en los migrantes haitianos, debido a que dominar esta lengua les permitirá tener mejoramiento económico, social y cultural. Lo anterior se ve reflejado en la Figura 2.6, donde el $72 \%$ de los migrantes desea conseguir un trabajo a través de un mejor dominio de la lengua; un $18 \%$, comunicarse y tener un mejor entendimiento con los ciudadanos de la frontera; un $5 \%$, conocer personas con el fin de establecer relaciones sentimentales; y el $5 \%$, sobrevivir en la ciudad para conseguir una residencia temporal hasta llegar a una residencia permanente.

\section{Me interesa hablar español para...}

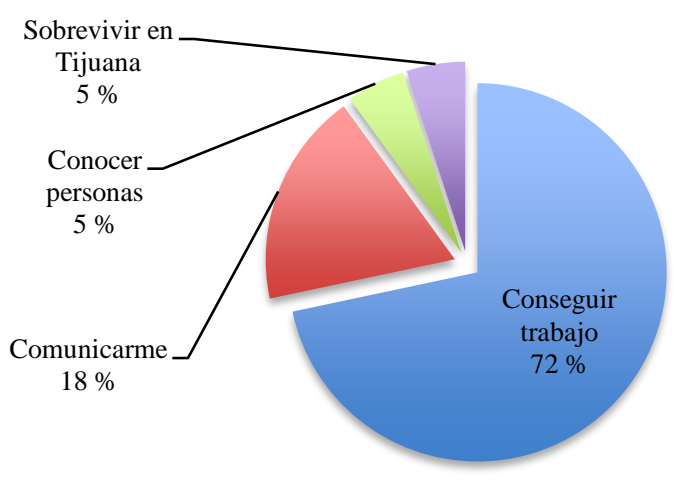

Figura 2.6. Intereses sobre el español

Para los nuevos migrantes, el inglés es una lengua de reto, ya que, para acceder a mejores escenarios laborales, este ocupa un lugar preponderante en la superación de quienes se mudan a Tijuana. Como se muestra en la Figura 2.7, para el $36 \%$ de los encuestados sus intereses de aprender inglés son aspirar a un mejor escenario laboral y obtener una visa humanitaria para vivir en Estados Unidos. El $31 \%$ utilizaría el inglés para conseguir un trabajo que sea mejor remunerado. Para un $31 \%$, el inglés representa una lengua de superación y, finalmente, un $2 \%$ ve el inglés como una lengua que les permitirá conocer personas para relacionarse y tener un mejor estilo de vida. 


\section{Me interesa hablar inglés para...}

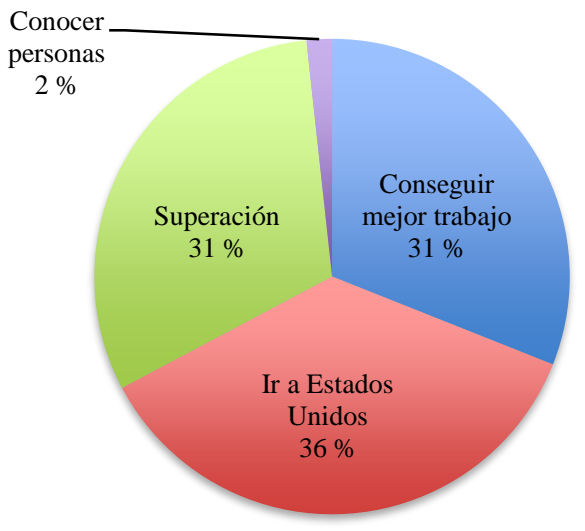

Figura 2.7. Intereses sobre el dominio del inglés

Por otro lado, existe un alto nivel de motivación con fines de permanecer en la frontera Tijuana-San Diego y el interés de esos nuevos migrantes o new comers es estudiar algún oficio en Tijuana, realizar estudios de educación básica y educación media superior. Para estos migrantes, dominar el idioma español les dará la oportunidad de realizar estudios en el sistema educativo mexicano. La Figura 2.8 muestra que a un $97 \%$ de los informantes les gustaría estudiar, con el fin de mejorar su estilo de vida y estatus económico. En contraposición con el $3 \%$, a quienes no les interesa realizar estudios.

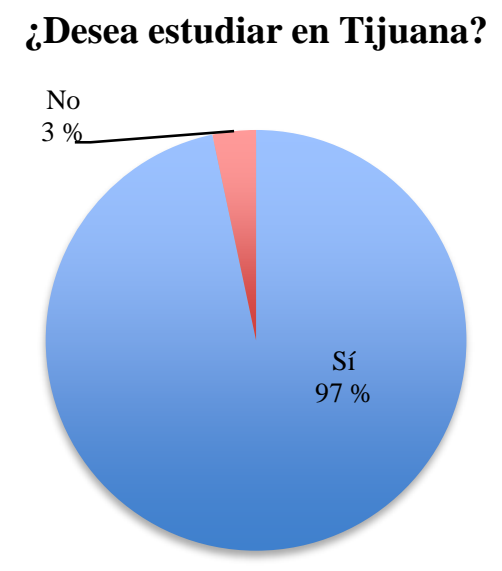

Figura 2.8. Intereses para realizar estudios en la frontera

Para los nuevos migrantes multilingües y centroamericanos, tener una oportunidad de permanencia les dará la opción de realizar estudios en territorio mexicano. En la Figura 2.9 se muestra que a un $20 \%$ le gustaría realizar estudios de posgrado, a un 
$20 \%$ realizar estudios de secundaria, es decir, que estos únicamente cuentan con estudios de educación básica. Un $17 \%$ tiene estudios de licenciatura y a un $13 \%$ le gustaría realizar una carrera técnica que les permita trabajar en oficios tales como: jardineros, cocineros, ayudantes de cocina, meseros y recepcionistas. Finalmente, un $17 \%$ no contestó este apartado donde se les preguntaba sobre el tipo de opciones de estudios que desean realizar. Se infiere que este porcentaje de migrantes no desea estudiar.

\section{Opciones de estudios}

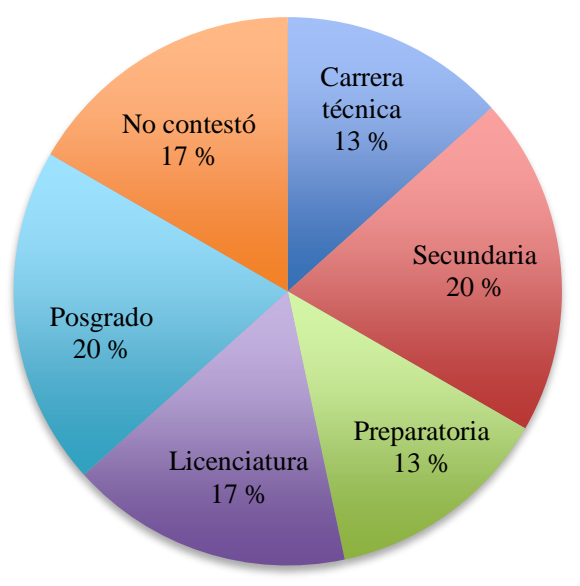

Figura 2.9. Opciones de estudios

Como se observó en los resultados obtenidos de la encuesta, se tiene un panorama visible de lo que esperan los migrantes al radicar en la frontera. Los escenarios lingüísticos emergentes en la frontera Tijuana-San Diego se determinan por aquellos nuevos migrantes o new comers que se han establecido en este espacio para vivir, trabajar, internarse en su cultura, pero no abandonar su lengua y su cultura. Por un lado, se encuentran los haitianos monolingües, quienes solo hablan criollo y aprenden español; por el otro, están los haitianos bilingües, quienes hablan criollo, portugués y aprenden español en proceso de ser multilingües. Los haitianos multilingües, quienes hablan criollo, portugués, español y aprenden inglés para tener una mejor oportunidad laboral, tienen el objetivo de ir en algún momento a radicar en los Estados Unidos. Este grupo se incorpora a actividades laborales en la región donde el idioma no es un limitante, debido al alto nivel de motivación, de sobrevivencia y resiliencia que tiene esta comunidad. Hablar español e inglés será un reto para insertarse en la dinámica social y cultural de la frontera. 
Es pertinente reflexionar sobre el uso de la lengua de los mexicanos retornados y deportados. Por un lado, están los migrantes quienes hablan un broken Spanish y poseen un mejor dominio del inglés, esto por el tiempo de haber radicado en Estados Unidos. Este motivo les permitió mejorar su nivel de inglés y perder su español. Otro grupo lo conforman los retornados o deportados que nunca aprendieron inglés inmersos en el contexto y que hablan la variedad lingüística Spanglish (mezcla de elementos léxicos y morfológicos), es decir, uso del inglés y el español sin tener conciencia de ello. Finalmente, se encuentra el grupo de migrantes monolingües que no aprendieron inglés y que su vida en Estados Unidos fue en contexto de negación hacia el aprendizaje del inglés.

Según Fundación BBVA (2013), con base en los resultados del estudio del perfil socioeconómico y de inserción laboral de mexicanos en retorno, la mayoría de los retornados son hombres casados, con familias construidas en Estados Unidos, con niveles educativos bajos, pero con un nivel de conocimiento del inglés aceptable. Estas personas se reincorporan a dinámicas de trabajo de servicios, manufactura, turismo y comercio. Este grupo de migrantes retornados se convierten en un grupo vulnerable, con condiciones de trabajo precarias, lo cual significa que la incorporación social al contexto mexicano será muy lenta. Al regresar a México harán uso de conocimientos para el trabajo y de sus habilidades lingüísticas (inglés) para demostrar que pueden conseguir un empleo, utilizando la lengua como herramienta de desarrollo económico.

De acuerdo con el estudio de Ramírez y Uribe (2013), los migrantes retornados regresarán a sus localidades rurales y a los lugares que conforman la región tradicional de migración en México para incorporarse a actividades del campo o a las ciudades donde existan oportunidades laborales. De hecho, Tijuana, por ser ciudad fronteriza y por su dinámica económica, es una zona propicia para que los migrantes retornados permanezcan y, aunque con dificultad, podrán integrarse al mercado de trabajo, esto gracias a sus destrezas y experiencias laborales y lingüísticas, tanto en el sector industrial como en el de servicios.

Obtener un empleo mejor remunerado, donde el inglés es un valor agregado, les dará apertura para obtener una seguridad social (servicio de salud, pensión, derecho a una vivienda digna, entre otros). 


\section{Conclusiones}

En este estudio se constata el papel que juegan los escenarios lingüísticos emergentes en la frontera Tijuana-San Diego, donde el ciudadano fronterizo bilingüe, llamado borderlander, vive inmerso en un proceso bilingüe que es determinado a partir de su experiencia con ambas lenguas: lo que escucha y lee en inglés y español en un ambiente real de inmersión.

Para fines de este estudio, se define a ese nuevo borderlander como el new comer criollo que, a partir de los nuevos desplazamientos migratorios, aprende español, aprende inglés para sobrevivir en la frontera y utiliza el portugués en caso de ser necesario para fines comunicativos entre la comunidad de habla criollo. Este es el nuevo migrante que se establece en Tijuana y formará parte del mosaico lingüístico y multicultural de una ciudad de migrantes.

También se definió al new comer multilingüe como el migrante haitiano que habla criollo, español, portugués y aprende inglés. No obstante, el dominio de varias lenguas y por el hecho de vivir en la frontera lo convierte en un borderlander multilingüe.

A la vez se describen los tipos de ciudadanos que radican en esta frontera. Por un lado, según Toledo Sarracino y Leopoldino (2018), un ciudadano que vive en la frontera es un new comer, pues existe, aprende, interactúa y experimenta el inglés y el español, y ambas lenguas están estrechamente vinculadas, donde la sociedad bilingüe nace y el multiculturalismo es parte de su cotidianeidad. Sin embargo, para los fines de este estudio, esta definición está mejor descrita con el surgimiento del nuevo ciudadano multilingüe que ha llegado a Tijuana.

La vida en la frontera se ve reflejada por la cotidianidad, pero también por el contacto de lenguas que se enlazan y hacen del inglés y el español lenguas fronterizas. Es decir, esta comunidad haitiana se convierte en el new comer fronterizo multilinguie que se desplaza por la zona fronteriza como la nueva tribu que, lejos de afectar, impacta de manera benéfica en la inserción laboral de las comunidades, en la creación de nuevas familias transfronterizas en Tijuana y la incorporación de una ciudad multilingüe.

Por otro lado, en esta frontera, se empieza a ver el desplazamiento por toda la ciudad del nuevo fronterizo multilingüe: el haitiano, establecido en la ciudad y que atraviesa por dificultades para permanecer en México y vivir en esta frontera en víspera de ingresar a los Estados Unidos con una visa humanitaria. Este proceso está muy alejado 
de la nueva realidad de migración en la frontera. Los haitianos han llegado como new comers para quedarse, expandir su cultura, su lengua, sus tradiciones, relacionarse y radicar en el país con un estatus migratorio de residente en México.

Dentro de este estudio, también se destaca el papel que juegan los migrantes retornados o deportados, quienes forman parte de esta realidad en la frontera. Estos connacionales que viven en Tijuana de forma temporal son considerados una población flotante, pero necesitan estar cerca del territorio norteamericano debido a que tienen familia en este lugar.

Según Peláez y París (2013), las personas que son deportadas se establecen temporalmente en Tijuana $\mathrm{u}$ otra ciudad en la frontera, con el fin de estar cerca de sus familiares en los Estados Unidos. Esto les permite sobrevivir, ganar dinero para costearse al "pollero" y prepararse para volver a cruzar. Las condiciones de vida son precarias, no se adaptan al entorno y no portan documentos de identidad ni certificados de estudios, mucho menos pruebas de experiencia laboral. Bajo esta situación, será difícil la incorporación a una sociedad mexicana, sin embargo, con el apoyo de organizaciones no gubernamentales y otras instancias de apoyo al migrante pueden lograrlo. El inglés será un valor agregado para obtener un mejor salario en el sector industrial y de servicios.

A partir de lo expuesto, se integrará una reflexión general sobre las implicaciones sociales, políticas, económicas, culturales, lingüísticas y educativas de la migración emergente en la frontera Tijuana-San Diego. De tal forma que la migración en el norte de México brinda un mosaico de información latente para el push-up de la frontera.

De acuerdo con los resultados obtenidos del cuestionario, para el estudio de escenarios lingüísticos emergentes en la frontera Tijuana-San Diego, se comprenden las nuevas dinámicas de migración en el desarrollo económico, social y la nueva integración de un mosaico lingüístico en la frontera donde se integra el criollo, el portugués, el español, el inglés y las lenguas originarias.

Para responder a la pregunta: ¿Qué experimenta esta zona fronteriza a partir de migraciones masivas de forma acelerada? Se considera que Tijuana vive una dinámica de combinación cultural y laboral con una integración de haitianos a fuentes de trabajo donde compiten en igualdad de circunstancias con aquellos retornados o deportados para buscar un trabajo. En ambos grupos existe un proceso de incorporación y adaptación: para los haitianos, la barrera del idioma, apoyarse mutuamente como nueva tribu urbana, permanecer y sobrevivir en un espacio fronterizo. Este grupo está dispuesto a permanecer, 
ya que las condiciones de vida son mejores que las que tenían en su país de origen. Por otro lado, los retornados, repatriados y deportados enfrentan ese shock cultural de regresar a México. Se enfrentan a nuevas dinámicas de la vida cotidiana, con carencias y limitantes sociales, económicas y políticas hasta incorporarse lentamente a una dinámica que es su realidad.

Se hace mención a que estas comunidades de migrantes lejos de tener limitantes para adaptarse a una nueva vida en México, tienen un valor agregado: haber vivido en un país donde se habla inglés, hablar la lengua y haber aprendido una cultura distinta a la propia, lo cual lo que lo hará un ciudadano competitivo en caso de que potencialice la lengua como su recurso para tener mejor oportunidades laborales.

Asimismo, Tijuana, por ser la frontera más visitada en el mundo y la ciudad más importante del noreste de México, tiene particularidades que otras fronteras del mundo no tienen. La migración hace que se tenga una diversidad lingüística; lo cual responde la siguiente pregunta: ¿Cómo se describe el mosaico lingüístico en esta frontera a partir de la nueva migración? A partir de los resultados de este estudio, se visualiza que la frontera Tijuana-San Diego tendrá un mosaico lingüístico distinto a otras fronteras por la mezcla de culturas asiáticas, americanas, centroamericanas y caribeñas. Ante ello, se señalan las siguientes comunidades: la comunidad haitiana que aprende español e inglés para tener mejores oportunidades de vida. Una realidad de las comunidades emergentes en esta frontera es que seguirá habiendo migración de haitianos, lo que significa que llegarán a Tijuana más new comers multilingües.

Según Wilner Metelus, presidente del Comité Ciudadano en defensa de los naturalizados y afromexicanos, la migración haitiana continuará en los siguientes meses. Llegan 250 diarios y el flujo continuará, dado que existen alrededor de 8000 varados en países como Panamá, Costa Rica, Nicaragua y Guatemala. México debe establecer medidas que facilite el apoyo a estos migrantes. Con certeza, los migrantes ven en la frontera el último lugar para llegar y establecerse en busca de una vida mejor. Es tarea de los gobiernos estatales y federales establecer estrategias para apoyar esta crisis migratoria. Ciertamente, se amplía el abanico de lenguas en contacto existentes en esta frontera, agregando el criollo y el portugués como lenguas adicionales al español e inglés.

Otro aspecto a considerar son los deportados y retornados. Según Gallardo (2017), se espera una deportación masiva de más de 50000 mexicanos por las medidas estrictas del gobierno de Donald J. Trump durante el 2017 y el resto del 2018. Esto significa que el gobierno mexicano, a través de sus programas de desarrollo social, debe apoyar a los 
migrantes en su inserción al sector laboral y escolar, así como establecer una política de apoyo a través de la Secretaría de Educación en los estados para que sean reconocidos los estudios de los migrantes en México. Aunque esto parezca una buena alternativa, se tendría que pensar en agilizar los procesos burocráticos para el reconocimiento, revalidación y acreditación de estudios en el extranjero. Esto es vigente también para los migrantes caribeños que poseen estudios $y$, en cierta medida, al permanecer en México desean realizar estudios en el sistema educativo mexicano, tal como se indica en este estudio.

En cuanto a la pregunta: ¿Cuál es el futuro de la política y planificación lingüística de esta frontera a partir de este crecimiento acelerado de migrantes? Se debe integrar en gran medida la labor en el desarrollo de investigaciones sociolingüísticas de la Universidad Autónoma de Baja California [UABC], la Universidad Nacional Autónoma de México [UNAM], el Colegio de la Frontera Norte [COLEF] y organizaciones no gubernamentales para planear el futuro de la política lingüística en esta frontera. Lo anterior permitirá coadyuvar al mejoramiento del mosaico lingüístico establecido en este espacio geopolítico. Es decir, integrar un equipo de especialistas que planifiquen el papel que juegan las lenguas en la frontera y brindar a los Gobiernos argumentos para la toma de decisiones en materia de políticas del lenguaje.

Según Calvet y Calvet (2013) y Bergenholtz y Tarp (s. f.), la diversidad linguiística en México es el resultado de la creación, aplicación y evaluación de política lingüística con base en el proteccionismo lingüístico en la frontera. El proteccionismo lingüístico está orientado a proteger una lengua contra la pérdida del domino y la reducción de su número de hablantes; hay iniciativas que establecen que el desarrollo lingüístico de los hablantes se estimule en la escuelas. Por su parte, México también tiene sus propias iniciativas, cuya prioridad es proteger las lenguas que se hablan y las que se integran en el abanico lingüístico. Es prioridad de las instituciones como la UABC, la UNAM y el COLEF aportar estudios serios sobre la diversidad lingüística y las lenguas emergentes y mecanismos de apoyo que se establezcan en Baja California.

Dicho lo anterior, ofrecer a través de las organizaciones no gubernamentales y la $\mathrm{UABC}$ un programa de enseñanza del español para migrantes. En la misma línea, es necesario buscar estrategias para reconocer y validar el nivel de inglés con base en estándares internacionales o exámenes internacionales para que el migrante retornado certifique su nivel de inglés. Esto le permitirá tener un respaldo que acredite la 
competencia comunicativa y obtener un certificado que avale dicho conocimiento. Baja California no ha realizado tareas al respecto en comparación con la Ciudad de México.

Gallardo (2017) señala que el Gobierno de la Ciudad de México y el Consejo Ciudadano A. C. son un ejemplo sobre el avance para certificar el inglés de retornados. Ambas instituciones apoyan a los mexicanos retornados para que puedan certificar el nivel de inglés con el examen Test of English for International Communication [TOEIC]. Esta certificación brinda al retornado o deportado una credencial lingüística para laboral y utilizar el inglés como valor agregado.

En sí, los migrantes retornados son un recurso humano habilitado en dos lenguas, quienes podrían formarse como docentes de idiomas en las universidades y normales mexicanas para impartir idiomas en la educación básica y superior, dependiendo de su nivel de estudios y de su conocimiento lingüístico. Baja California debe seguir este modelo y apoyar a estos migrantes para que tengan el reconocimiento del nivel de inglés en la frontera. Con certeza, reconocer los estudios de los retornados o deportados y establecer el nivel de idioma permitirá que se incorporen al sector laboral mejores ciudadanos bilingües.

Finalmente, se concluye que los resultados obtenidos de este trabajo permitieron comprender el dinamismo de la migración en el desarrollo social, económico y cultural de la frontera Tijuana-San Diego, que da a este espacio un crecimiento demográfico de la población migrante en el norte de México. Asimismo, facilita visualizar el futuro de las lenguas en espacios fronterizos a partir de las migraciones emergentes y reubicar aquellas políticas que se han quedado al margen en el pasado, así como idear otras nuevas que puedan dar respuesta a las problemáticas expuestas en esta investigación. 


\section{Referencias bibliográficas}

Bergenholtz, H. y Tarp, S. (s. f.). Política linguiística: Conceptos y definiciones. Canadian Translators, Terminologist and Interpreters Council. Recuperado de http://www.cttic.org/ACTI/2004/papers/Henning_Bergenholtz_y_Sven_Tarp_P oliticalinguistica.pdf. Consulta: 08 de junio de 2017.

Calvet, Louis-Jean y Calvet, Alain. (2013). Les confettis de Babel. Diversité linguistique et politique des langues. Montréal: Écriture.

COLEF. (2016). Proyecto Archivo con testimonios de los migrantes del Colegio de la Frontera Norte [COLEF]. Recuperado de https://www.colef.mx/noticia/arribantijuana-mexicali-mas-hombres-haitianos-africanos-mujeres/. Consulta: 23 de julio de 2017.

Couza, Dalia. (2014). De migración, migrantes, deportados y retornados. Efecto migrante. Enlace México. Recuperado de https://www.colef.mx/saladeprensa/wpcontent/uploads/2014/02/Dolo1.jpg. Consulta: 23 de julio de 2017.

Donnan, Hastings y Wilson, Thomas. (1999). Border, Frontiers of identities. Nations and State. Oxford: Berg.

Durand, Jorge. (2000). “Tres premisas para entender y explicar la migración MéxicoEstados Unidos". Relaciones. Estudios de historia y sociedad, XXI(83). Recuperado de http://www.redalyc.org/articulo.oa?id=13708302. Consulta: 25 de julio de 2017.

El Universal. (2017). Comité Ciudadano en Defensa de los Naturalizados y $\begin{array}{lll}\text { Afromexicanos } & 2017 . & \text { Recuperado de }\end{array}$ http://www.eluniversal.com.mx/articulo/estados/2016/10/11/denuncianxenofobia-africanos-y-haitianos-en-redes-sociales. Consulta: 23 de julio de 2017.

Fundación BBVA. (2013). Estudio del perfil socioeconómico y de inserción laboral delos migrantes mexicanos en retorno. Ciudad de México: BBVA.

Gallardo, Constanza. (2017). A new beginning is always possible for these deported immigrants. Recuperado de www.aristeguinoticias.com. Consulta: 19 de junio de 2017.

García-Landa, Laura. (2017). (En dictamen). "Lo mejor de Tijuana es San Diego", "lo mejor de San Diego es Tijuana" construcción metafórica de identidades corporeizadas en la inter-transacción entre dos lenguas-culturas. En Sabine 
Pfleger (ed.), Somos en el lenguaje y a través de él. El lenguaje como sistema complejo en el estudio de complejidades sociales. México: UNAM.

Ibarra González, Israel. (2016). Inserción laboral y trayectoria de los migrantes mexicanos en Tijuana. Tesis de Maestría en Desarrollo regional. Recuperado de https://www.colef.mx/posgrado/wp-content/uploads/2016/11/TESIS-IbarraGonz\%C3\%A1lez-Jos\%C3\%A9-Israel.pdf. Consulta: 28 de julio de 2017.

Institute of Naturalization Services. (2017). Non inmigrant policies. Recuperado de https://www.uscis.gov/policymanual/HTML/PolicyManual-Volume2.html. Consulta: 17 de mayo de 2017.

Instituto Nacional de Lenguas Indígenas. (2008). Catálogo de las lenguas Indígenas Nacionales: Variantes Lingüísticas de México con sus autodenominaciones y referencias geoestadísticas. Recuperado de http://www.inali.gob.mx/pdf/CLIN_completo.pdf. Consulta: 28 de julio de 2017. Instituto Nacional de Lenguas Indígenas. (2015). Informes de mexicanos deportados. http://www.inali.gob.mx/es/institucional/mision-y-vision.html. Consulta: 01 de noviembre de 2016.

Loza, Lourdes. (2017). Persiste interés por obtener el asilo político. Recuperado de http://www.frontera.info/EdicionEnLinea/Notas/Noticias/16052017/1215027-

Persiste-interes-por-obtener-el-asilo-politico.html. Consulta: 17 de mayo de 2017.

Morris Bermúdez, R. (2007). Al borde del multiculturalismo: Evaluación de la política lingüística del estado Mexicano en torno a sus comunidades indígenas. Recuperado de http://confines.mty.itesm.mx/articulos5/MorrisR.pdf. Consulta: 28 de julio de 2017.

Peláez, Diana y París, María Dolores. (2013). Mujeres deportadas en Tijuana: Separación familiar y sentimientos de exilio. Recuperado de https://www.colef.mx/emif/resultados/articulos/2013\%20$\% 20$ Mujeres\%20deportadas\%20en\%20Tijuana\%20separacion\%20familiar\%20y \%20sentimientos\%20de\%20exilio.pdf. Consulta: 28 de julio de 2017.

Ramírez, Telésforo y Uribe, Luz María. (2013). El retorno de migrantes mexicanos desde Estados Unidos: ¿Un fenómeno individual o familia? Ciudad de México: CONAPO.

Smith, Patrick. (2006). Transnacionalismo, bilingüismo y planificación del lenguaje. En Roland Terborg y Laura García-Landa (coords.), Los retos de la planificación del lenguaje en el siglo XXI, 2. México: UNAM. 
Terborg, Roland et al. (2006). "The Language Situation in Mexico". Current Issues in Language Planning, 7(4), 415-518.

Toledo Sarracino, D. G. y Leopoldino, Gabriel. (2018). La política lingüística en espacios fronterizos: una reflexión contrastiva México-Uruguay. En Daniel Rodríguez y Joaquín Martínez (coords), Exploración de principios de práctica actuales en laenseñanza y aprendizaje de lenguas. México:UNAM.

\section{(9) $\odot \Theta \Theta$}

Esta obra está bajo una licencia de Creative Commons Reconocimiento-NoComercial-SinObraDerivada $\underline{4.0 \text { Internacional }}$ 